\title{
Construction of the Network Learning Platform for the Course Building Space Transformation based on Grid
}

\author{
https://doi.org/10.3991/ijet.v13i05.8437 \\ Chen Zhu \\ Army Logistics University of PLA, Chongqing, China \\ zhuchen $1101 @ 163 . \mathrm{com}$
}

\begin{abstract}
It is a development trend to build a network learning platform for the current university course teaching, but universities or colleges can't share the learning information with each other effectively. Meanwhile, the whole screen synchronous display will consume wireless network mobile data. This network learning platform can combine the grid with the network synchronization technology to synchronize the information displayed on the multimedia equipment with the learners' computers. Besides, it can share the network instructional resources. A new teaching technology combining the course synchronous system with the network learning platform proposed in this article with the grid, the network teaching system and the synchronization technology of network learning platform as the theoretical basis is composed of 2 parts: the learning module (including the course learning function and the assignment uploading and correcting function) and the synchronous display module. We tested the practical effect of the new network learning platform and found that this platform could broaden the learning space of the architecture specialty, facilitate the sharing of learning resources, improve the learning quality effectively, and complement the implementation of traditional learning methods beneficially.
\end{abstract}

Keywords-Grid, network learning platform, building space transformation

\section{Introduction}

The birth of the computer has improved the computing function and the computational efficiency, and the development of computer technology lies in the information exchange, which is also the root cause for the generation of internet. If the information cannot be exchanged, each individual computer cannot be connected with each other, which is also the basis for the information isolated island theory [1]. Therefore, the generation of internet is to eliminate the information isolated island generated by the individual computer, to share the information resources, and to unify the information exchange at both software and hardware levels. The existence of internet has proved the necessity of information sharing very well. Different information can be shared and disseminated effectively through internet. 
To learn the modern educational technology, the development of the network learning platform is a trend at present, which not only includes sharing the corresponding hardware, but also includes sharing the learning information. It is a new direction for the development of the current learning mode. The teaching efficiency of the whole network learning platform can be improved effectively by sharing the corresponding resources. Therefore, the corresponding network learning platforms have been researched at home and abroad for sharing information and improving the efficiency [2].

In this article, we chose the network learning platform based on grid as the main research object. The advantages of the network learning platform are to improve the quality and effect of offline learning, and to transmit and share the learning resources efficiently. Moreover, all course content can be displayed through the rich multimedia functions [3] on the network learning platform abundantly and flexibly, so that students can participate in learning in different forms and with different methods to improve their interest and understanding in learning.

\section{State of the art}

The network learning platform [4] is revised and designed based on Dokeos Open Source System, which is a comprehensive teaching service support system including online teaching, teaching coaching, online self-learning, online training for new employees and other services to provide the real-time and non-real-time teaching coaching services for students and enterprises, and to help managers to control various learning content, record learners' learning state and progress, and arrange various learning activities and learners' learning process. Generally, it has the following functional modules: flexible course management, learning record trace analysis, grouping management of classes and groups, course resources management, etc. With the rapid development of internet, the scale of network learning platforms is being expanded gradually, and the governments of all countries begin to pay attention to the network learning platforms gradually. At present, most universities and colleges in China take their campus networks as the infrastructure for the network learning platform to provide an advanced network computing environment for all teachers and postgraduates in universities and colleges and all scientific research personnel in scientific research institutions [5]. Usually, the campus networks of universities and colleges not only provide various network services such as WWW, Mail, FTP, video on demand and database retrieval of journals and papers, but also provide many management services such as education, teaching, scientific research and teaching affairs at the same time. For example, Zhang put forward to combine the cloud computing with the campus network intelligent platform of universities and colleges organically to share the information and the campus network data, and to further improve the utilization rate of the campus network intelligent learning platform [6]. Chen introduced that we could build a wireless network with twisted-pair quickly based on the original wired campus network, so as to complete the design and use of the wireless campus network. Besides, after a long period of online operation, it is proved that the wireless network 
learning platform in this university was scientific, fast and stable [7]. However, due to the lack of teaching resources in accordance with distance education standards and interscholastic cooperation, the problem of repeated development is prominent, and a lot of manpower and material resources have been wasted. The phenomenon of critical shortage of resources in campus networks is widespread, and there is no rounded system structure and specific implementation to share resources really [8]. Theoretically, the education grid can solve the above problems completely, but the premise is that the universities and colleges and the institutions engaged in distance education shall establish their own grid teaching nodes at first.

At present, the grid is researched mainly in USA and Europe. The government of Britain has invested GBP 100 million for the development of the UK National Grid [9]. USA is planning to implement a grand grid plan called Global Information Grid, which is expected to be completed in 2020 [10]. With the accelerated research on the grid in the academic field, many large companies in the information industry have published their R\&D plans consistent with the network goals. HP, IBM, Microsoft, Sun and other companies have reached a consensus to support XML, SOAP, UDDI and other Web standards, so as to more conducive to the development of the new generation of network applications, namely, Web services [11].

With the gradual development of multimedia technology, the learning mode of network learning platform has been applied to the architecture specialty gradually. At present, the network learning platform has some shortcomings. The main shortcoming is that the learning information cannot be shared effectively between schools. Meanwhile, for the current synchronous display of the software screen applied to the network learning platforms, the whole screen is refreshed, captured and transmitted regularly, which will consume the wireless mobile data easily, result in the phenomenon such as picture lag, non-fluency, and low regular operating efficiency. Especially for learning the architecture specialty, the practical ability is required highly, so the inappropriate learning methods may increase students' learning pressure and the complexity of learning.

For above reasons, we proposed a new synchronization technology of network learning platform at the same time in this article, whose innovation and advantages are: (1) it can combine the teachers' teaching equipment with the students' learning equipment effectively and organically, to share the corresponding information resources through the exchanger of internet or between the local area networks. Meanwhile, this new synchronization technology can meet the teaching requirements for the whole architecture and civil engineering course effectively and improve the teaching efficiency of the architecture course. (2) Combined with a network synchronization technology, this network learning platform can not only synchronize the information displayed on the multimedia equipment with the learners' computers, but also display the corresponding content displayed on computers on the teachers' multimedia platform in real time. The advantages of this technology are to reduce the utilization rate of the hardware in the multimedia equipment effectively and improve the transmission speed of corresponding multimedia resources the service life of hardware. 


\section{Model analysis}

\subsection{Grid and network teaching system}

Grid is an important information technology emerged in the world in recent years, which can organize the computers scattered in different geographical locations to be a "virtual supercomputer" by internet [11]. Besides, it is the organic combination and development of high-performance computers, data sources and internet. The characteristics of grid are distribution and sharing, multi-layered isomerism of system, unpredictability of structure, and high service quality (including response time, flow, usability and security). The grid system applied to this research is a five-layered sand clock structure. Namely, it is a structure with wide influence. The system distributes the functions of operating, managing and using the shared resources into five different layers according to the distance between each component and the shared resource, emphasizes the "protocols", and mainly pays attention to the qualitative description. The structure is as shown in Fig.1:

\section{grid computing protocol | Internet protocol}

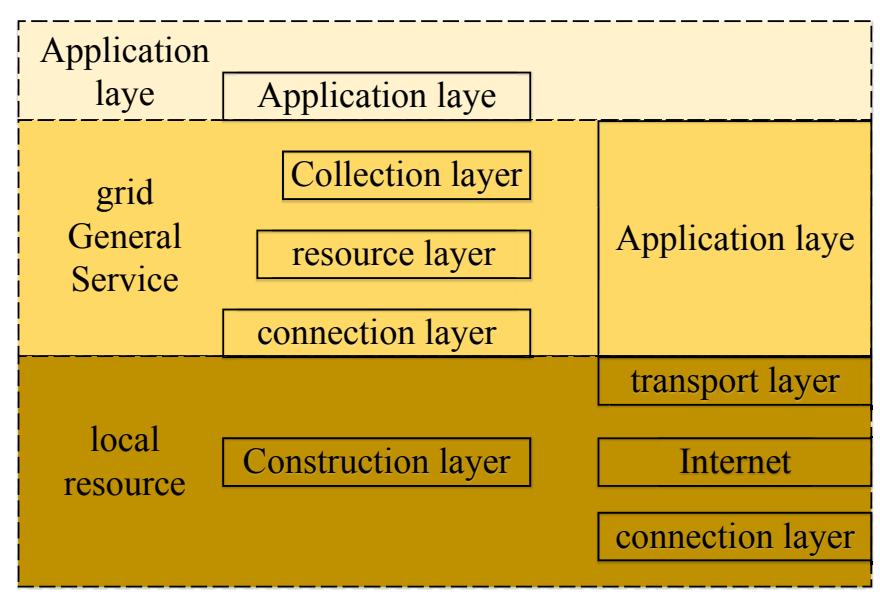

Fig. 1. The sketch of the five-layered sand clock structure of the grid on the network learning platform

Another important feature of the five-layered structure is its shape like a sand clock, which means that the quantity of protocol in each part is different. For the core part, all protocols on the upper layer shall be mapped to the core protocol. Meanwhile, the core protocol shall be mapped to all kinds of protocols on the lower layer. The core protocol shall be supported at all sites supporting the grid computing. In the fivelayered structure, the resource layer and the connection layer constitute the bottleneck of the core as shown in Fig.2. 


\begin{tabular}{|c|c|}
\hline Tool and application & application layer \\
\hline $\begin{array}{c}\text { diagnosis and } \\
\text { monitoring }\end{array}$ & $\begin{array}{c}\text { Resource and connection } \\
\text { layer }\end{array}$ \\
\hline $\begin{array}{c}\text { Secure access to } \\
\text { resources and services }\end{array}$ & Tectonic layer \\
\hline various resources: computers, & \\
\hline
\end{tabular}

Fig. 2. The five-layered sand clock structure

The network learning platform proposed in this article combines the grid and the network synchronization technology well. After the combination, integrate the learning characteristics of the architecture specialty with its content to form a network learning platform with corresponding characteristics. By the network teaching system mentioned in this article, on the one hand, the content of the architecture specialty can be displayed in diversified forms; on the other hand, the experiment content designed in the course of architecture specialty can be combined with the practical engineering effectively to form a new network learning platform.

\subsection{The synchronization technology of network learning platform}

The most important function of the synchronization technology of network learning platform is to synchronize the teaching operations on other platforms with the multimedia equipment used by students effectively. This technology mainly depends on the information transmission function of the campus network so as to transmit the corresponding teaching information in real time. The synchronization technology of network learning platform mainly includes the following modules:

The detection module. The teachers detecting the network learning platform can conduct the corresponding operations on the computer terminal platform, transfer the corresponding information to the information processing terminal of the server after processing and packaging, and then display the information synchronously allowed by the information processing terminal.

The information processing module. In the information processing step, it is needed to judge whether the information fed back in the detection step is reasonable. Meanwhile, it is needed to judge the teachers' behavior changes in the teaching process. If the result of the information processing is positive, the information processing module will package the processed information and send it to the output module to display the final picture. The formulas for the synchronous transmission image output are Formula (1) and Formula (2) to determine the locations of the images.

$$
\begin{aligned}
& x^{\prime}=x \cos \theta-y \sin \theta \\
& y^{\prime}=y \cos \theta+x \sin \theta
\end{aligned}
$$


The output module. The main function of the output module is to receive the picture information transmitted by the information processing module and display it on the information screen terminal. The output module shall be connected with the network transmission equipment to transmit the information transmitted by the information processing module to the multimedia terminal for students in class by internet or the internal local area network, so that teachers and students can be connected with each other face to face.

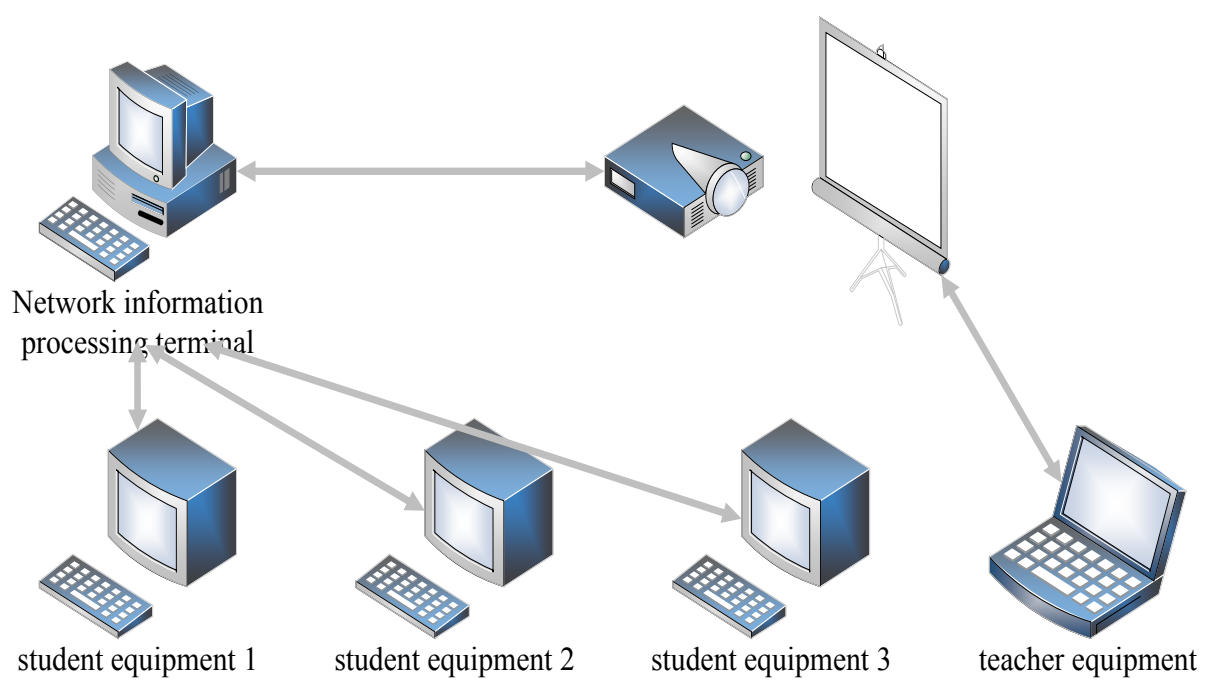

Fig. 3. The basic operation structure chart of the network synchronization technology

\section{The synchronization technology of network learning platform in learning the course Building Space Transformation}

\subsection{The overall framework of the network learning platform based on grid}

The network learning platform designed in this article shall not only own the functions for the traditional network learning, but also combine the network learning synchronization technology with the grid technology organically, to form a new network learning platform meeting the requirements of the course Building Space Transformation. In addition to the basic functions of the learning module, this platform has the function of synchronous display module for online learning.

Fig.4 is the distance education structure chart based on the campus grid. On the server side, a grid operating environment is required at first. After the teaching grid service is developed, it shall be deployed into the operating environment. As the grid service for the background operation, it can be separated from the server providing the user interface and supporting Java, because the grid service can be located anywhere in the grid system. The user interface is a webpage made with Servlet十Html. 
On this page, the administrator can create and manage management domains, and learners can register to log into the grid teaching system and learn in the corresponding management domains. Once the system receives a request for learning, the web server will call the grid service by Servlet, and then transmit it to the user interface in the form of HTML.

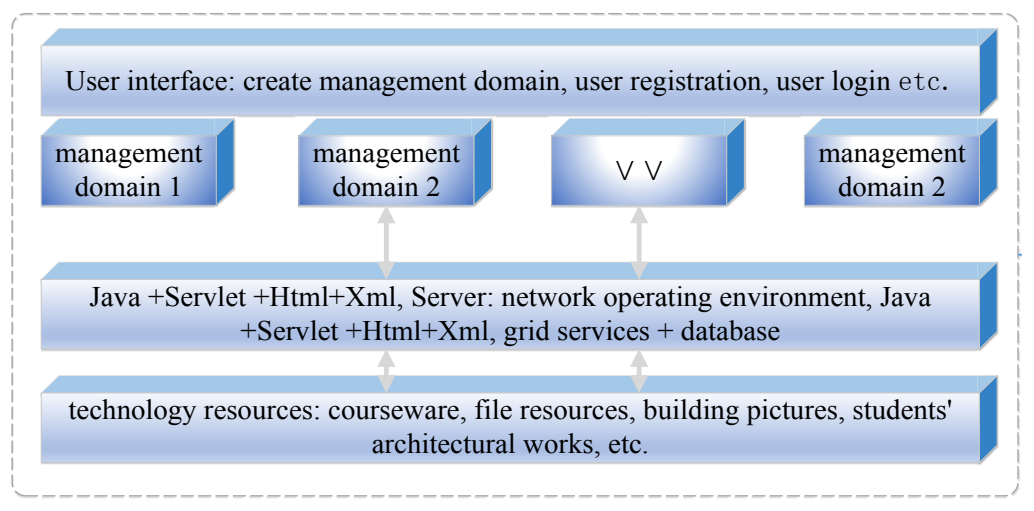

Fig. 4. The distance education structure chart based on the campus grid

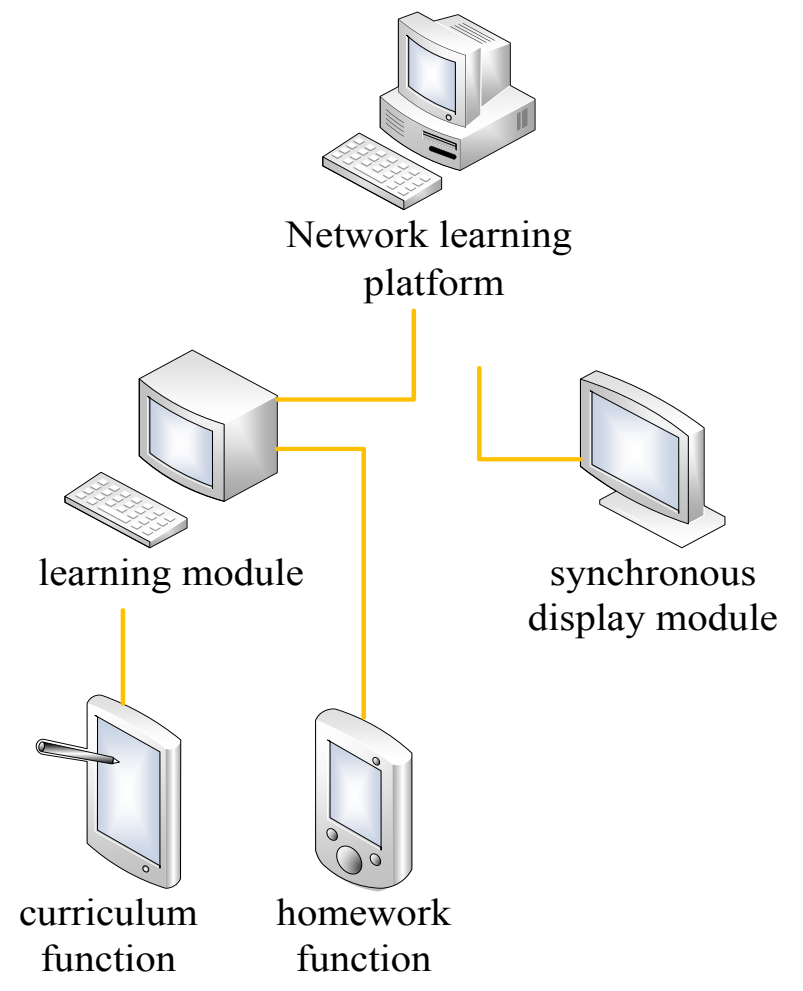

Fig. 5. The function structure chart of the network learning platform 


\subsection{Realize the functions of the network teaching system based on grid}

The learning module. The learning module is the most important content of this network learning platform, whose main feature is to make students learn in the network learning platform and experience all online and offline learning functions in teaching and learning processes. The online learning module mainly contains the following functions.

The network learning platform in this research has published the specific information of the course, Building Space Transformation, including the course content, subjects, reference data and other relevant information resources required in the teaching process. Students can effectively complete the preview and review of learning with the information provided by the course function of the learning module.

In the course, Building Space Transformation, teachers will not only design the building space transformation accordingly, but also divide the whole building space design into several small design modules, because the design workload of overall building space is huge, and the task is heavy. These small design modules also will be intended to be the assignments of the course by teachers, so the assignment quality is various and large. The functions to upload and correct assignments shall be stable in this network learning platform.

In Fig.6, the step to upload the assignment of the course, Building Space Transformation, is displayed in the networking teaching system based on grid.

The synchronous display module. The features of the synchronous display module in this network learning platform are to capture teachers' movements on the network teaching platform and display the actual building space model on the students' multimedia equipment synchronously with the synchronization technology of network learning platform. The actual effect picture displayed on the students' multimedia equipment is shown in Fig.7.

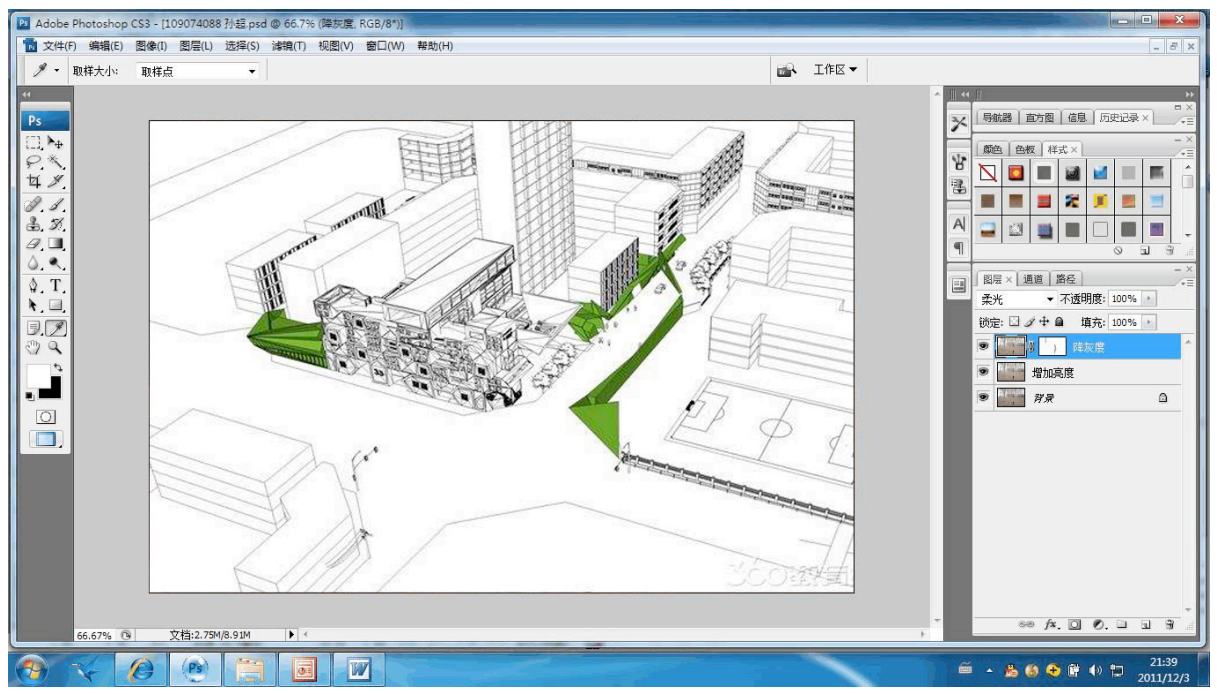

Fig. 6. The assignment uploading module 


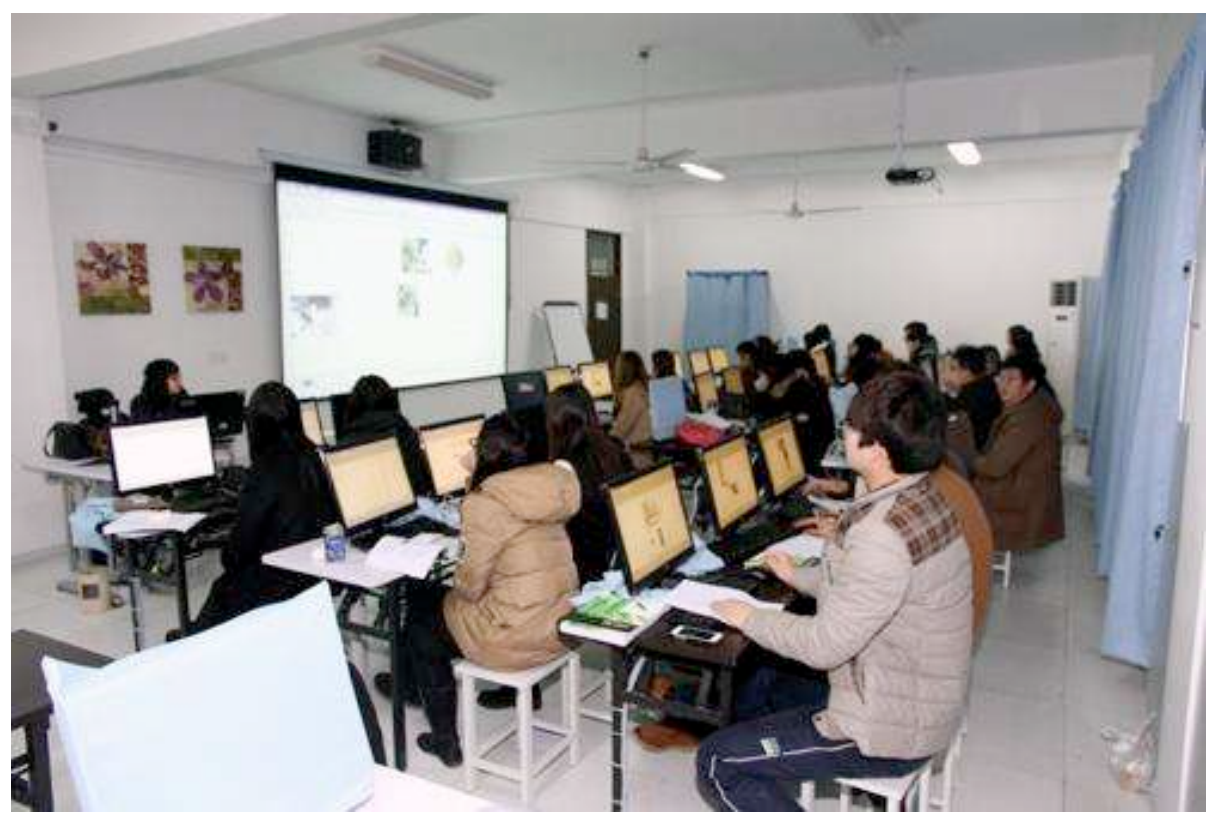

Fig. 7. Display the network teaching system based on grid

Test the teaching effect. We had a contrastive analysis of 150 students participating in learning the Building Space Transformation. The research period was from September 2016 to December 2016. Among them, there were 72 students using the network learning platform based on the grid, and 78 students using the ordinary network learning system. The students in the experimental group experienced the learning with this learning platform tentatively for ten lessons. Each lesson was for 120min. After learning, we mainly analyzed the students' evaluation on the difficulty in learning and their academic records. There was no difference between the teachers of the two groups in the teaching level, and no difference between the students of the two groups in their academic records before the experiment.

After 10 times of learning experience, we had a statistic of the students' evaluation on the difficulty of the experiment as shown in Table 1.

We had a statistics of the final theory scores and experiment scores of the students in the two groups as shown in Table 2:

By the contrastive analysis of the two groups of the students learning the Building Space Transformation, we can discover that the network learning platform based on grid put forward in this article could improve students' learning effect greatly. It is obvious from Table 1 that the students in the experimental group learned the course more easily, and this system could lower the difficulty of new knowledge for students. In addition, Table 2 shows that teachers evaluated the overall learning state of the students in the experimental group higher, and the total scores of the experimental group were higher than that of the control group. 
Paper-Construction of the Network Learning Platform for the Course Building Space Transforma..

Table 1. The comparison of students in the two groups on the evaluation of the knowledge difficulty

\begin{tabular}{|l|c|c|c|c|c|}
\hline \multicolumn{1}{|c|}{ Difficulty } & Number of students & Easy & Medium & Difficult & Very difficult \\
\hline Experimental group & 72 & 35 & 34 & 21 & 10 \\
\hline Control group & 78 & 26 & 29 & 33 & 22 \\
\hline
\end{tabular}

Table 2. The final comparison of the academic records between the two groups $(\bar{x} \pm s)$

\begin{tabular}{|l|c|c|c|c|c|}
\hline \multicolumn{1}{|c|}{ Item } & $\begin{array}{c}\text { Theory } \\
\text { scores }\end{array}$ & $\begin{array}{c}\text { Experiment } \\
\text { scores }\end{array}$ & Defense scores & Group scoring & Total scores \\
\hline Experimental group (72) & $8.54 \pm 0.57$ & $8.57 \pm 0.52$ & $8.47 \pm 0.33$ & $8.76 \pm 0.56$ & $43.5 \pm 10.33$ \\
\hline Control group (78) & $7.79 \pm 0.34$ & $7.96 \pm 0.53$ & $8.19 \pm 0.23$ & $8.68 \pm 0.62$ & $36.3 \pm 19.1$ \\
\hline T value & 4.348 & 3.879 & 0.597 & -1.687 & 2.456 \\
\hline $\mathrm{P}$ & 0.000 & 0.000 & 0.513 & 0.063 & 0.000 \\
\hline
\end{tabular}

\section{Conclusions}

We find that the new network learning platform based on grid have better teaching effect and learning effect. The primary reason is the teaching mode: while the students were applying the new network learning platform, teachers could modify the teaching mode in normal times according to the advantages of the system and the application features. Especially they combined the common theoretical knowledge with the course experimental design effectively to form a practice-oriented teaching mode for the architecture course. This teaching mode can help students to learn in practice to get a better effect than the students learning theory first and practicing later in the past. Moreover, it is helpful to supervise students' learning and increase the interaction between teachers and students. For example, students can participate in learning actively with the network teaching platform. Teachers can improve, modify or delete modules, update knowledge, expand new content, and increase the utilization of resources in time by looking over the visiting rate of the network learning platform, browsing the learning section and the browsing time and according to students' needs for learning. In addition, teachers can adjust the learning tasks for students appropriately to play the role of supervised learning by accessing the record of students' autonomous learning status.

The learning platform based on grid is integrated with the network synchronization technology innovative, and it has been combined and applied to the learning of architecture specialty. After the application of this network learning platform based on grid, we found that this learning facility based on the campus network and highperformance computing technology has a good application prospect. It can broaden the learning space of architecture specialty, facilitate the sharing of learning resources, improve the learning quality effectively, and complement the implementation of traditional learning methods beneficially. Although there are many problems to be solved in the research on grid, the grid will certainly become a network computing model connecting and unifying all different long-distance resources. In this article, we 
just took much theoretical discussion and analysis, and we still need some specific projects for practice and verification. In addition, due to the lack of time and the researchers' limited knowledge capability, we didn't conduct a deep research on other courses and problems, which is the theme we will further pay attention to.

\section{References}

[1] Lenzner, B., Weigelt, P., Kreft, H., et al. The general dynamic model of island biogeography revisited at the level of major flowering plant families. Journal of biogeography, 2017, vol. 44(5), pp. 1029-1040. https://doi.org/10.1111/jbi.12906

[2] Terkowsky, C., Pleul, C., Jahnke, I., \& Tekkaya, A. E. Tele-Operated Laboratories for Online Production Engineering Education - Platform for E-Learning and Telemetric Experimentation (PeTEX). Global Engineering Education Conference, 2011, vol. 6, pp. 491497.

[3] Nee, A.Y.C. A review of the application of grid technology in manufacturing. International Journal of Production Research, 2011, vol. 49(13), pp. 4119-4155. https://doi.org/10.1080/ 00207541003801234

[4] Virtanen, S. Increasing the self-study effort of higher education engineering students with an online learning platform. International Journal of Knowledge \& Learning, 2008, vol. 4(6), pp. 527-538. https://doi.org/10.1504/IJKL.2008.022886

[5] Yang, K.X., Cao, Q.X. Analysis of Campus Network Platform Research and Teaching Software Integration. Computer CD Software and Applications, 2015, vol. 2, pp. 64-66.

[6] Zhang, X. University Network Intelligent Learning Platform Based on Cloud Computing. China Science and Technology Information, 2017, vol. 9, pp. 47-48.

[7] Chen, W.F. Design and Implementation of Local University Wireless Campus Network Based on WLAN. Network Security Technology \& Application, 2017, vol. 8, pp. 108-110.

[8] Zhang, P., Wang, J.Z., Zhou, T.M. Research on secure examination system with immune capacity based on education grid. Journal of Computer Applications, 2006, vol. 26(2), pp. 349-351.

[9] Hardman, S., Chandan, A., Shiu, E., et al. Consumer attitudes to fuel cell vehicles post trial in the United Kingdom. International Journal of Hydrogen Energy, 2016, vol. 41(15), pp. 6171-6179.

[10] Fernandez, T. Technological and economic comparison of battery technologies for USA electric grid stabilization applications, Doctoral dissertation: Massachusetts Institute of Technology, 2010.

[11] Allcock, B., Foster, I., Nefedova, V., et al. High-performance remote access to climate simulation data: a challenge problem for data grid technologies. Parallel Computing, 2003, vol. 29(10), pp. 1335-1356.

\section{$7 \quad$ Author}

Chen Zhu is a Postgraduate of Army Logistics University of PLA, Chongqing 401311, China. His research interests include Camp Planning and Design. (zhuchen1101@163.com).

Article submitted 13 February 2018. Resubmitted 18 March 2018. Final acceptance 25 March 2018. Final version published as submitted by the author. 